Article

\title{
The Functional Resonance Analysis approach to assess performance variability during emergency conditions
}

\author{
Antonella Petrillo ${ }^{1}$, Luigi Ranieri ${ }^{2}$, Laura Petrillo ${ }^{3}$ and Fabio De Felice ${ }^{4, *}$ \\ 1 Department of Engineering, University of Napoli “Parthenope”, Isola C4, Centro Direzionale 80143 Napoli, \\ Italia; antonella.petrillo@uniparthenope.it \\ 2 Dipartimento di Ingegneria dell'Innovazione, University of Salento, Centro Ecotekne Pal. O - S.P. 6, Lecce - \\ Monteroni, Italia; luigi.ranieri@unisalento.it \\ 3 Dipartimento di Ingegneria Civile e Meccanica, University of Cassino and Southern Lazio, via G. Di Biasio \\ 43, 03043 Cassino (FR), Italia; defelice@unicas.it \\ 4 S.C. Prevenzione e Sicurezza Ambienti di Lavoro S.S. PSAL Porto: Ponte Andrea Doria 16126 Genova, Italia; \\ laurapetrillo1@virgilio.it \\ * Correspondence: antonella.petrillo@uniparthenope.it
}

\begin{abstract}
Technological innovation has led to the development of increasingly efficient and complex industrial plants. To manage this complexity, it is necessary to define an integrated vision of the socio-technological system that includes: technological, human and organizational component. Petrochemicals can be considered one of the most complex socio-technical systems that deserve special attention to high risk management, especially during the emergency conditions. Traditional safety management models only consider static systems, while new resilience engineering models evaluate the performance variability developed between different actions. One of the recent development methods is the Functional Resonance Analysis Method (FRAM) that identifies the pairs between the functions. FRAM unfortunately is a qualitative model, this research integrates this model with the Performance Shaping Factors (PSFs) and with the Bayesian approach to identify the performance variability of the system. The analysis aims to develop a system that improves safety analysis. The proposed model is applied in a case study of an emergency in a petrochemical company.
\end{abstract}

Keywords: human reliability analysis; safety; FRAM; resilience engineering; performance variability; emergency

\section{Introduction}

Although over the years, industrial plants have improved their safety management processes, we all agree that safety systems need to be further improved. This need is underlined by the many accidents that have occurred in industrial plants over recent years, arising from human causes, technical causes or natural causes. Traditional safety management models are designed to identify negative factors and develop systems to mitigate their impact.

Several systems use this approach. For example, Petrillo et al. (2017) [1] analyze the emergency conditions in a petrochemical company through the Human Reliability Analysis (HRA), which analyzes the human errors that have caused the emergency and it calculates the HEP. The Hazard and Operability analysis (HAZOP) identifies accidental events and related problems with a logical cause of the process parameters. Casamirra et al. (2007)[2] use HAZOP to develop a risk analysis of the storage unit in a hydrogen refuelling station. The Failure Mode Effect and Critical Analysis (FMECA) model analyzes individual components and their failures. The analysis is based on the definition of a risk index that classifies the failure probability. Mechhoud et al. (2016) [3] use FMECA for risk analysis and assessment in petrochemical plants. In some cases hybrid models are also generated, for example Giardina and Morale (2015) [5], integrated FMECA and HAZOP to study a regasification plant. Zhou et al., (2017) [5] define a new model called "D-DEMATEL" to identify the 
critical success factors in emergency management. This model integrates the number theory with the decision making. Ramzali et al., (2015) [6] use the Event Tree Analysis (ETA) to define a safety barrier analysis of the offshore drilling system. The ETA is a failure modeling technique that explores the probability of the outcomes by building a tree. The Failure Tree Analysis (FTA) is an error analysis in which a failure is analyzed using a boolean tree logic. Akgun et al., (2015) [7] use FTA to determine the optimal positions of supply plants to be used during emergency management.

Although these models allow to analyze different critical situations, they seem ineffective for today's business needs. Particularly in modern industrial plants, only a few functions are independent of each other, so analyzing them individually may not be the best model. In petrochemical systems, as in other socio-technical systems, it is not possible to identify all functions in a specific way, but it is necessary to identify flexible macro-functions. In this perspective, the resilience engineering says that we should not consider the two-mode system as operating or malfunction, but related to daily performance and their variability, which could generate success or failure. Resilience engineering refers to very complex systems, so it is difficult to measure specific results. Hollnagel (2012) [8] defines complex systems through their functions and studies the interactions between these functions through the Functional Resonance Analysis Model (FRAM). This document develops a quantitative model, integrating traditional FRAM with the environmental performance evaluation.

The proposed model overcomes the qualitative limits of the resilience engineering models proposed in the literature, furthermore through the integration of the PSFs it allows to create a direct relationship between the variability of performance and the influence of the external environment, furthermore using Bayesian model, the research define a correlation between time and accuracy of the activity. The model is extremely flexible and can be applied in different applications. It is a decision support tool that helps managers manage complex systems.

The rest of the paper is organized as follows. Section 2 analyzes the state of art on FRAM. Section 3 describes the framework of the proposed model. Section 4 presents a case study in a petrochemical industry and its results. Finally, section 5 summarizes the conclusion of the research and the future developments.

\section{Materials and Methods}

\subsection{Resiliecence Engineering}

Resilience engineering describes the characteristic features of complex systems and looks for ways to improve the organization's ability to create robust and flexible processes, to monitor and review risk models and to use proactive resources in the face of unexpected developments [9]. This literature review section, first it describes a bibliographic analysis of resilience engineering models, after which it focuses on the FRAM by analyzing the application fields and hybrid FRAM. The literature analysis was carried out on the Scopus database. The bibliographic analysis was developed through the use of different keywords. The first research is related to the keyword "resilience engineering". The database has identified 250 publications with this keyword. The first publications are quite recent, dating back to 2005, but in recent years there has been a very high increase. This demonstrates the growing interest in these issues, especially in the USA, Brazil and Iran, which are at the top three places as interested researchers on these topics. In particular, the University of Teheran is in first place as publications on this topic. Research subjects are related to engineering, computer science and social sciences. $50 \%$ of publications are articles, while $30 \%$ are conference papers. One of the most used resilience engineering models is the FRAM. For this reason, the research previously carried out has been extended using two different keywords linked to the Boolean operator of "and" intersection.

The search string is: "resilience engineering" and "FRAM". In this case the result of the research shows 37 publications that have the 2 defined keywords. The publications are concentrated in the last 5 years and have a fairly high growth rate. In this case the most "productive" nations are Brazil, Sweden and Denmark. Also, in this case the number of articles is greater than the number of 
conference papers. An interesting application of the resilience engineering models is for the evaluation of the management of complex systems in emergency situations.

Thus, the third search keyword is "emergency". This keyword was added to the other two and the database returned 12 publications, including 11 articles and 1 conference paper. In this case, Sweden, Brazil and Italy are the three most "productive" nations. Analyzing the 12 publications, it is clear that, there are several literature reviews and several case studies. Cross-analysis of citations shows that the literature review articles are much more cited than in case studies. The 3 most cited articles are described below:

- Rankin et al. (2014) [10] develop a framework for the analysis of high-risk jobs in complex systems through the resilience engineering approach;

- Righi et al. (2015) [11] describe a systematic literature review of resilience engineering considering 237 studies developed between the 2006 and the 2014;

- Bergstrom et al. (2015) [12] develops a bibliographic research about the resilience engineering, with particular attention to the safety implications.

Figure 1 shows the trend of the number publications in the various years based on the analyzed keywords.

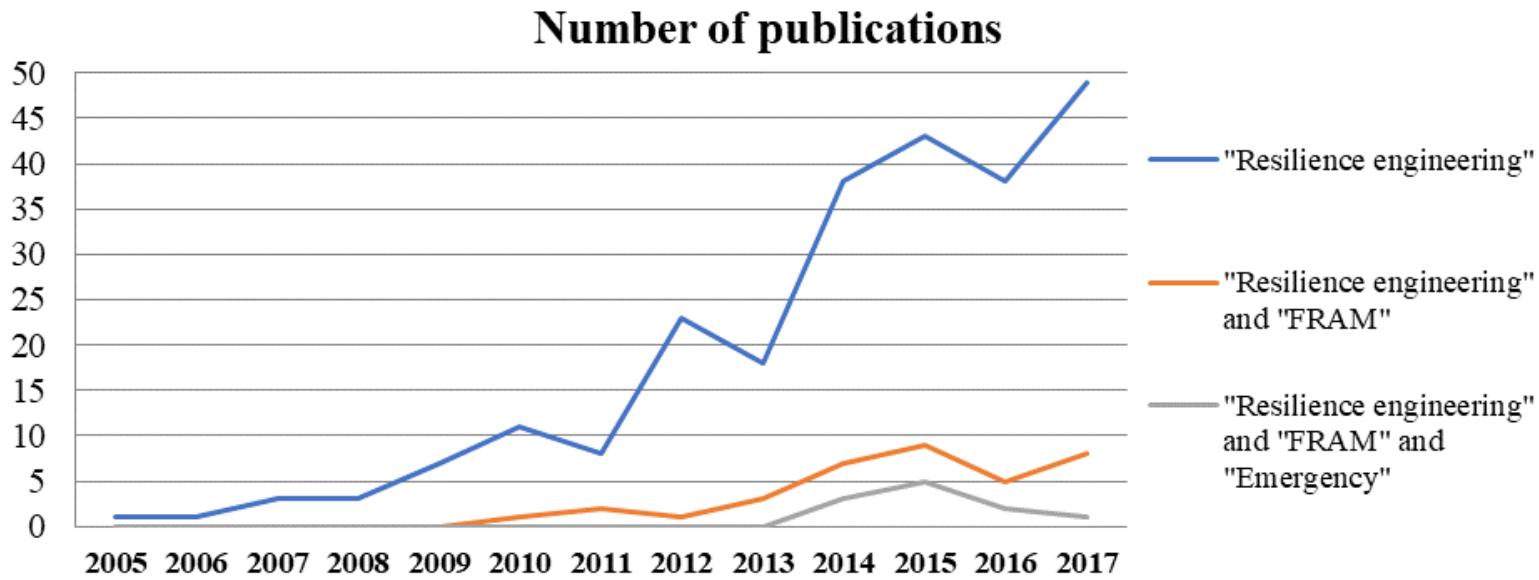

Figure 1. Number of publications.

The subsequent literature analysis focused on the FRAM and in particular on the application of this model and on the development of hybrid FRAM developed through integration with other scientific tools in order to overcome the limitations of the traditional FRAM. Table 1 shows the various fields of application of the FRAM resulting from literature analysis.

Table 1. Fields of application FRAM.

\begin{tabular}{|c|c|c|c|c|c|c|c|}
\hline \multirow[b]{2}{*}{ Authors } & \multicolumn{7}{|c|}{ Field of application FRAM } \\
\hline & Aeronautic & Space & Nuclear & $\begin{array}{c}\text { Health } \\
\text { care }\end{array}$ & Railway & Oil & Sea \\
\hline Sawaragi et al. (2006) & $\checkmark$ & & & & & & \\
\hline Hollangel et al. (2008) & $\checkmark$ & & & & & & \\
\hline Herrera et al. (2010) & $\checkmark$ & & & & & & \\
\hline Patriarca et al. (2016) & & $\sqrt{ }$ & & & & & \\
\hline Lundblad et al., (2008) & & & $\checkmark$ & & & & \\
\hline Shirali et al. (2013) & & & & & & $\checkmark$ & \\
\hline Sujan e Felici (2012) & & & & $\checkmark$ & & & \\
\hline Pereira (2013) & & & & $\checkmark$ & & & \\
\hline
\end{tabular}


Steen e Aven (2011)

Belmonte et al. (2011)

Tian et al. (2016)

Hollnagel (2012)

$\checkmark$

$\checkmark$

The FRAM methodology allows to manage systems considering the order among the various activities that compose them and considering how a single upstream activity can influence downstream activities. The methodology was born and developed in the aeronautical field for the management of systems with a view to reliability and safety, but it is used in many other sectors. Sawaragi et al., (2006) [13] systematically analyze the effects of automation under variable conditions in the pilot cabin of an aircraft. Hollangel et al. (2008) [14] analyze the Comair Airlines flight accident in 2006 in Lexington. Herrera et al., (2010) [15] use the FRAM to analyze safety performance indicators for offshore helicopter traffic. Patriarca et al., (2016) [16] apply the FRAM for the resilience analysis of a space mission, with particular attention to safety aspects. Another application of the FRAM is analytical for industrial systems, particularly those at high risk such as nuclear power plants. Lundblad e Speziali (2008) [17] apply the FRAM for the qualitative assessment of risks in lifting an 80-tonne fuel container at a nuclear power plant. Also, in other industrial fields the FRAM is applied, for example Shirali et al., (2013) [18] identify emerging risks in an Iranian oil company. Sujane and Felici (2012) [19] use the FRAM method as a complement to the FMEA model to assess socio-technical risks in the health care industry. Pereira (2013) [20] applies the FRAM for the risk assessment of a pharmaceutical radio dispatch process, highlighting possible critical situations. Hollnagel (2012) [8] analyzes through the FRAM a surgical incident in which the surgical material remained involuntarily in the patient's abdomen. Steen and Aven (2011) [21] analyze with the FRAM an analysis of the system risks related to potential cyber physical attacks on the railway network, causing a collision between two trains. Belmonte et al., (2011) [22] show the advantages deriving from the adoption of the FRAM for the analysis of railway traffic safety. Tian et al., (2016) [23] use the FRAM for risk analysis and performance variability on ferry accidents at sea.

The major problem of the FRAM is its extremely qualitative characteristic. To overcome this gap, several FRAM, integrated with other quantitative scientific instruments, have been developed (Table 2). Bjerga et al., (2016) [24] analyzes FRAM in terms of uncertainty modeling, showing the need to integrate its context with other reliable decision-making approaches. The most important model, described in literature, is presented below.

Rosa et al., (2015) [25] and De Carvalho (2011) [26] uses the FRAM integrated with the Analytic Hierarchy Process to reduce subjectivity related to performance variability. Zheng et al., (2016) [27] combine the FRAM with the SPIN model to verify different variability paths. Praetorius et al., (2016) [28] they combine FRAM with Formal safety assessment (FSA), a structured methodology in the decision-making process of maritime safety. Patriarca et al. (2017) [29] define a theoretical semi-quantitative FRAM to evaluate the variability of functions by integrating the traditional FRAM with the Monte Carlo simulation. The proposed model has been applied for semiquantitative evaluation in a sintering plant [30].

Table 2. Hybrid FRAM.

\begin{tabular}{cccccc}
\hline Authors & \multicolumn{4}{c}{ Hybrid FRAM } \\
\cline { 2 - 5 } & AHP & SPIN & FSA & Montecarlo & FMEA \\
Rosa et al., (2015) & $\checkmark$ & & & & \\
De Carvalho (2011) & $\checkmark$ & & & & \\
Zheng et al., (2016) & & $\checkmark$ & & & \\
Praetorius et al., (2016) & & & $\checkmark$ & & $\checkmark$ \\
Patriarca et al., (2017a) & & & & $\checkmark$ & \\
Sujan e Felici (2012) & & & & & \\
\hline
\end{tabular}


All the models described do not consider the influence of the external environment on the variability of performance and the correlation between time and accuracy activity. The research model that will be presented considers the numerical influence of the external environment considering different PSFs and the weight of each PSFs in relation to the analyzed activity, furthermore, it uses Bayesian mathematical approach to consider the mathematical correlation between time and accuracy of the activity.

\subsection{Research model}

The model presented below uses an integrated FRAM approach that allows to define the probability of occurrence of particular instances, based on the environmental conditions of the system, on working time and integrating, through a Bayesian approach, the dependence between distinct events. The basic structure is defined by the FRAM proposed by Hollnagel (2012) [8], a detailed analysis of environmental factors is implemented considering the research proposed by Gertman et al., (2005) [31]. Environmental factors are weighed by the experts of the field. Moreover, considering the research of Patriarca et al., (2017) [28] each instance is represented by a probability of occurrence and in this study the dependencies between different variables are also considered through the Bayesian model. The structure of the hybrid model is shown in Figure 2.

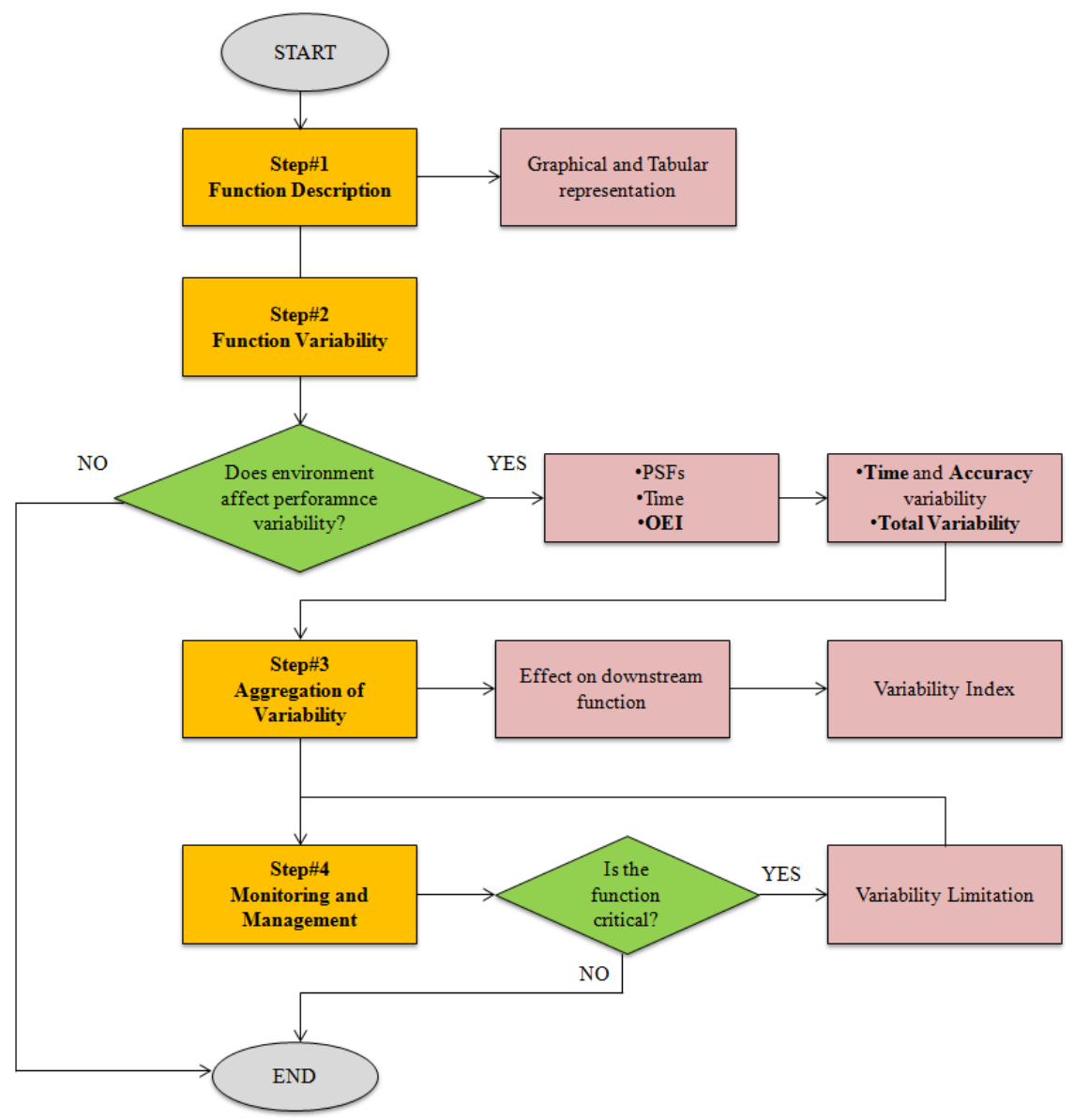

Figure 2. Methodological approach flowchart.

\subsubsection{Step \#1: Function description}

It is necessary to describe the goal of the analysis and the daily actions (functions) necessary to perform the activities analyzed. FRAM analysis starts from a function and develops by adding iteratively any other function that may be necessary to provide a complete description of the system. In the FRAM the connections or couplings between the functions are well defined. The functions are represented as a hexagon (Figure 3) with the following 6 different characteristics: 
- Input (I): function activation. The inputs can be more than one. The foreground functions must have inputs, while the background functions may not have them;

- Output (O): result of a function. The output can be considered as a change in system status or one or more parameters. The output is fundamental to explain how the variability of a function can propagate in the system;

- Precondition (P): preconditions that must exist for a function to be performed;

- Resource (R): condition of execution of the function;

- Control (C): monitoring of the function in order to produce the desidered output;

- Time $(\mathrm{T})$ : temporal constraints that influence the function.

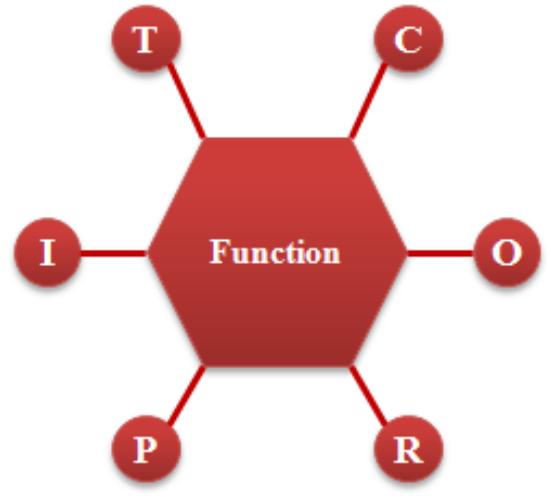

Figure 3. FRAM hexagon.

The six elements described allow us to understand how performance can vary and how variability can be combined to produce unexpected out-of-scale problems. In addition to the hexagonal graphical representation, each function can be represented by the tabular form (Table 3).

Table 3. Function description.

\begin{tabular}{lc}
\hline & Function name \\
\hline Aspects & Description \\
Input & \\
Output & \\
Precondition & \\
Resource & \\
Control & \\
Time & \\
\hline
\end{tabular}

In the FRAM conception, there are no orders, the model consists of a set of functions description. The functions are potentially coupled if they have common descriptions. So even if the output of a function can be combined with the precondition function of another, this does not mean that it will always be executed before the other. The FRAM analyzes how the potential links of a model can become real pairs in a model instance.

\subsubsection{Step \#2: Function variability}

Performance variability is the fundamental element of FRAM. If the output of a function does not change, then the variability of the function is of no interest, while it becomes interesting a function that has an output variability and therefore could generate a variability on the downstream functions. Variability may depend on different elements and causes. The FRAM describes the possible coupling between the functions and describes the variability of the potential of the functions. The potential variability describes what could happen if the circumstances are right or 
wrong. Actual variability describes what should realistically be expected under certain conditions, needs, opportunities and resources.

Thus, if the operating conditions (e.g. temperature) for the technology are well within the design limits, then the potential variability is not likely to become effective. For human functions, if the activities take place in a shift work environment, it is reasonable to suppose that there will be an actual variability due to the effects previously described. In this case it is reasonable to assume that the potential variability will be expressed as actual variability.

From the age of the study of human reliability, environmental factors are considered a source of performance variability. Gertman, et al. (2005) [31] describe various environmental factors that could affect the performance of a system. The idea behind this semi-qualitative FRAM is to describe the environment in which the operator is working and quantifying this influence. Based on the scenario analyzed for each factor, a multiplier is defined (Table 4).

Table 4. Performance Shaping Factors.

\begin{tabular}{ccc}
\hline Factors & Level & Values $(\boldsymbol{\alpha})$ \\
\hline \multirow{3}{*}{ Time } & Inadequate & 5 \\
& Medium & 0.1 \\
& High & 0.01 \\
Stress & Extreme & 5 \\
& High & 2 \\
Complexity & Nominale & 1 \\
& Very Complex & 5 \\
& Moderately Complex & 2 \\
Training & Nominal & 1 \\
& Low & 3 \\
& Nominal & 1 \\
Procedures & High & 0.5 \\
& Not available & 50 \\
& Incomplete & 20 \\
& Poor & 5 \\
Ergonomics & Incomplete & 50 \\
& Poor & 10 \\
& Nominal & 1 \\
Process work & Nominal & 1 \\
& Good & 0.5 \\
\hline
\end{tabular}

Furthermore, many works in the literature such as Petrillo et al. (2017) [32] define the variability of human performance closely linked to working time. As time goes by the operator may vary (in negative) his performance.

Thus, for human and organizational analysis another factor to consider is relative to working time. Also, in this case, 4 different multipliers are defined according to the working time (Table 5). Furthermore, future research developments could increase work hours beyond 8 hours. 
Table 5. Time values.

\begin{tabular}{cc}
\hline Hours & Values $(\boldsymbol{\tau})$ \\
\hline 1 & 1 \\
2 & \\
3 & 2 \\
4 & \\
5 & 3 \\
6 & \\
7 & \\
8 & 4 \\
\hline
\end{tabular}

The quantification of environmental factors is very important, because it allows the quantitative translation of aspects that in general are always qualitative. To make the model stable, it is necessary to weigh the environmental factors, according to the function considered. The factor weights are established by experts during the analysis process.

After defining the values of multipliers that refer to environmental factors, their relative weights, and the working time, it is necessary to calculate the value of an overall environmental index (OEI). This index has been hypothesized to represent a unique value that describes the overall environmental representation.

The calculation of the index is shown below:

$$
O E I=\left(\sum_{i=1}^{n} \alpha_{i} \omega_{i}\right) \tau
$$

Where:

a_i it is the value of environmental multipliers;

$\omega \_i$ it is the weight of environmental multipliers;

$\tau \quad$ it is the value related to the working time.

Considering the OEI value, 3 different scenarios can be defined, each with a different external influence (Table 6).

Table 6. Different scenario.

\begin{tabular}{cc}
\hline Scenario & Total influence factor \\
\hline A. Low external influence & OEI $<7$ \\
B. Medium external influence & $7 \leq$ OEI $<20$ \\
C. High external influence & OEI $\geq 20$ \\
\hline
\end{tabular}

For each scenario defined as such, it will be possible to choose a value of variability of time and accuracy referred to a specific function, each with its own score (Table 7). 
Table 7. Performance variability.

\begin{tabular}{ccc}
\hline Output Variability of upstream function & Score (a) \\
\hline \multirow{3}{*}{ Time } & Too early & 2 \\
& In time & 1 \\
& Too late & 3 \\
& Omission & 4 \\
Accuracy & Inaccurate & 4 \\
& Acceptable & 2 \\
& Accurate & 1 \\
\hline
\end{tabular}

Also, according Patriarca et al. (2017) [29], for each of the three scenarios it is possible to define a probability of variation of output performance (Figures 4 and 5). The choice of probability depends on the evaluation of experts in the sector. For a given scenario, there may be different probabilities related to time variability (probT) or accuracy variability (probA).

Probability (time)

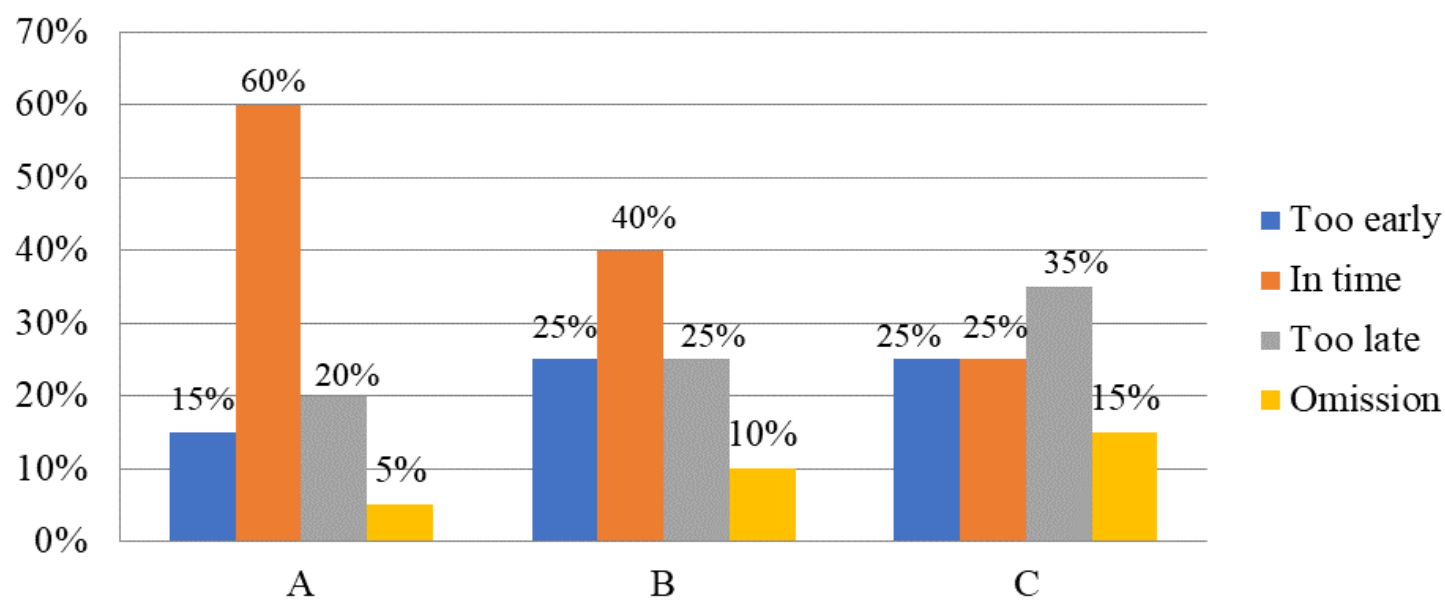

Figure 4. Probability of time variability.

\section{Probability (accuracy)}

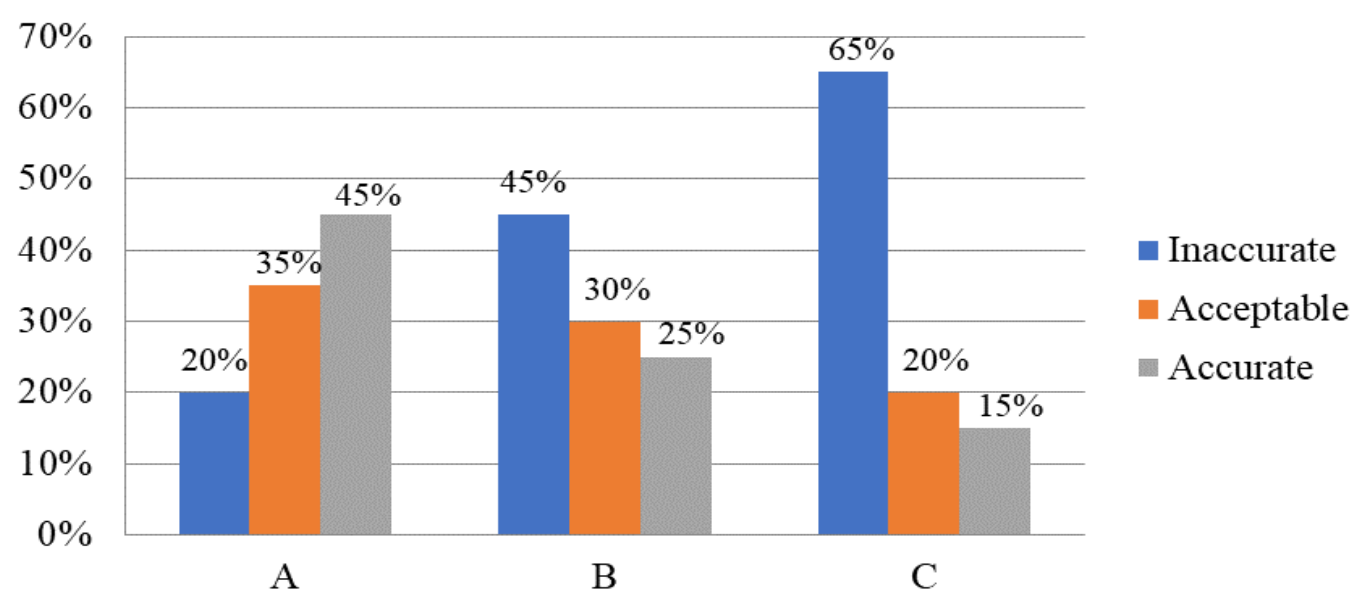

Figure 5. Probability of accuracy variability. 
Furthermore, it is fundamental to identify an interdependence factor between time variability and precision variability (Table 8). The choice is entrusted to the experts of the sector, the object of analysis.

Interdependence is very important to realize a real system, since often the time of realization of a function can influence its accuracy or vice versa.

Table 8. Dependence factor.

\begin{tabular}{cc}
\hline Dependence & Value (o) \\
\hline Very low & $10 \%$ \\
Low & $20 \%$ \\
Medium & $30 \%$ \\
High & $40 \%$ \\
Very high & $50 \%$ \\
\hline
\end{tabular}

If the time influences the accuracy, the combined probability can be calculated using the Bayes theorem:

$$
\operatorname{Prob}(\mathrm{A} / \mathrm{T})=[\operatorname{Prob}(\mathrm{T}) * \rho] / \operatorname{Prob}(\mathrm{A})
$$

If instead the accuracy influences the time it is possible to apply the Bayes theorem by inverting the variables:

$$
\operatorname{Prob}(\mathrm{T} / \mathrm{A})=[\operatorname{Prob}(\mathrm{A}) * \rho] / \operatorname{Prob}(\mathrm{T})
$$

The total variability of the function for a single scenario (TV) is calculated as the sum of the time variability and the accuracy variability multiplied by their probability values.

If time affects accuracy, then:

$$
T V=a^{T} \cdot P(T)+a^{A} \cdot P\left(\frac{A}{T}\right)
$$

If accuracy affects time, then:

$$
T V=a^{T} \cdot P\left(\frac{T}{A}\right)+a^{A} \cdot P(A)
$$

\subsubsection{Step \#3: Aggregation of variables}

It is not enough to evaluate the variability for the single function. It is necessary to understand how variability can be combined. This is achieved using the upstream-down functional coupling. The variability of the function can be the result of couplings of upstream functions that influence downstream functions. Each upstream variable can be connected to its downstream variable with the output and one of the 5 available inputs. Depending on the type of connection, different variability occurs (see tables below). 
Table 9. Aggregation of funtions (Output - Input).

\begin{tabular}{ccc}
\hline Output Variability of upstream function & Possible effects on downstream function \\
\hline \multirow{2}{*}{ Time } & Too early & Premature start $(\mathrm{V}+)$ \\
& In time & Possible damping $(\mathrm{V}-)$ \\
& Too late & Possible damping $(\mathrm{V}-)$ \\
& Omission & Delayed activities $(\mathrm{V}+)$ \\
& Inaccurate & Start imprecision $(\mathrm{V}+)$ \\
Accuracy & Acceptable & Waste of time $(\mathrm{V}+)$ \\
& Accurate & No change $(\mathrm{V}=)$ \\
& & Possible damping $(\mathrm{V}-)$ \\
\hline
\end{tabular}

Table 10. Aggregation of funtions (Output - Precondition).

\begin{tabular}{ccc}
\hline Output Variability of upstream function & Possible effects on downstream function \\
\hline \multirow{2}{*}{ Time } & Too early & False start $(\mathrm{V}+)$ \\
& In time & Possible damping $(\mathrm{V}-)$ \\
& Too late & Possible waste of time $(\mathrm{V}+)$ \\
& Omission & Possible waste of time $(\mathrm{V}+)$ \\
Accuracy & Inaccurate & Possible misunderstanding $(\mathrm{V}+)$ \\
& Acceptable & No change $(\mathrm{V}=)$ \\
& Accurate & Possible damping $(\mathrm{V}-)$ \\
\hline
\end{tabular}

Table 11: Aggregation of funtions (Output - Resources).

\begin{tabular}{|c|c|c|}
\hline Output Variability of upstream function & Possible effects on downstream function \\
\hline \multirow{4}{*}{ Tempo } & Too early & No change $(\mathrm{V}=)$ \\
\cline { 2 - 3 } & In time & Possible damping $(\mathrm{V}-)$ \\
\cline { 2 - 3 } & Too late & Possible damping $(\mathrm{V}-)$ \\
\cline { 2 - 3 } & Omission & Possible waste of time $(\mathrm{V}+)$ \\
\hline \multirow{4}{*}{ Precisione } & Inaccurate & Possible waste of time $(\mathrm{V}+)$ \\
\cline { 2 - 3 } & Acceptable & Operation reduction $(\mathrm{V}+)$ \\
\cline { 2 - 3 } & Accurate & Possible damping $(\mathrm{V}-)$ \\
\hline
\end{tabular}


Table 12: Aggregation of funtions (Output - Control).

\begin{tabular}{|c|c|c|}
\hline \multicolumn{2}{|c|}{ Output Variability of upstream function } & Possible effects on downstream function \\
\hline \multirow{4}{*}{ Time } & Too early & Missing control (V+) \\
\cline { 2 - 3 } & In time & Possible damping (V-) \\
\cline { 2 - 3 } & Too late & Control default $(\mathrm{V}+)$ \\
\cline { 2 - 3 } & Omission & Find substitute control (V+) \\
\hline \multirow{4}{*}{ Accuracy } & Inaccurate & Delays (V+) \\
\cline { 2 - 3 } & Acceptable & No change $(\mathrm{V}=)$ \\
\cline { 2 - 3 } & Accurate & Possible damping $(\mathrm{V}-)$ \\
\hline
\end{tabular}

Table 13: Aggregation of funtions (Output - Time).

\begin{tabular}{ccc}
\hline Output Variability of upstream function & Possible effects on downstream function \\
\hline \multirow{3}{*}{ Time } & Too early & Incorrect time $(\mathrm{V}+)$ \\
& In time & Possible damping $(\mathrm{V}-)$ \\
& Too late & Delays $(\mathrm{V}+)$ \\
& Omission & Start inaccuracy $(\mathrm{V}+)$ \\
Inaccurate & Amplification $(\mathrm{V}+)$ \\
& Acceptable & No change $(\mathrm{V}=)$ \\
& Accurate & Possible damping $(\mathrm{V}-)$ \\
\hline
\end{tabular}

After defining the couplings between the functions, it is necessary to calculate numerically the performance variability of the pair of functions analyzed. The numerical value of the effects (EF) between the upstream variable (i) and the downstream variable (j) is shown below:

$$
E F_{i, j}=\left\{\begin{array}{c}
2 \text { Amplification }(V+) \\
1 \text { No effect }(V=) \\
0.5 \text { Damping }(V-)
\end{array}\right.
$$

The amplification of the system has value 2, no effect has value 1, while the damping has a value of 0.5 . At this point the performance variability generated by an upstream variable on a downstream variable can be calculated as the product between the performance variability on the upstream function for the effects (related to time and accuracy) on the downstream variable.

$$
\mathrm{VAR}=\mathrm{VT} * \mathrm{EF}_{\mathrm{ij}}^{\mathrm{T}} * \mathrm{EF}_{\mathrm{ij}}^{\mathrm{A}}
$$

\subsubsection{Step \#4: Monitoring and Management}

The last phase of the model involves monitoring and managing any events of uncontrolled variability if they can lead to a negative risk or to undesired results. If this is possible you need to make sure that it does not happen. Likewise, variability can lead to desirable situations, in this case it is necessary to facilitate and improve the results. The purpose of the survey is to find critical 
combinations and reinforce the barriers. The problems of complex systems can not be eliminated, eliminating the variability of the performances, because this is essential to ensure the reliability of the systems. The solution is to manage performance variability by trying to dampen it to prevent resonance effects. It is necessary to fix a limit value called VAR* above which the function becomes critical and it is necessary to apply systems to limit uncontrolled variability (Table 14).

Table 14: VAR classification.

\begin{tabular}{cc}
\hline Acceptable & $\mathrm{VAR}^{*}<1$ \\
\hline Slightly critical & $1 \leq \mathrm{VAR}^{*} \leq 5$ \\
\hline Critical & $\mathrm{VAR}^{*}>5$ \\
\hline
\end{tabular}

Below are some solutions to be adopted when performance variability must be limited.

The classic solution is the elimination of risks, removing parts, components or activities of the system. Elimination is effective, but it is a rare event, as it may require the redesign of the whole system. Another solution is a prevention that requires a barrier or defense. Typically, the barriers are effective but expensive, it is necessary to find the right compromise. Facilitation is aimed at all that is useful, could involve changes in the system, to manage it optimally. Facilitation allows you to make activities less complex, doing the right things. Protection is focused on the results of an accident rather than on the events that generate it. Protection is a reactive, ineffective activity. Monitoring is essential to verify if the objectives are being achieved. Damping allows you to manage variability and control it.

\section{Theoretical model applied in a real case study}

The presented theoretical model was applied in a real case study: the management of an emergency in a petrochemical company. The plant consists of process and service plants as follows:

\section{Process plant:}

- Predistillation unit;

- Propane deasphalation unit;

- Distillation unit;

- Catalytic hydrogenation unit;

- Diesel oil purification.

\section{Service facilities:}

- Diathermic oil system;

- Steam and hot water production unit;

- Refinery torch;

- Hydrogen production unit;

- Cooling water system;

- Refinery storage area.

The plant preserves extremely dangerous substances in quantities equal to or greater than the limits, so it is a high risk activity, where it is necessary to analyze all the deviations from the operating standards (emergency conditions) such as: gas leakage, hydrocarbon release, fire, earthquake, flood, sabotage, pollution, etc. 


\section{Results}

\subsection{Step \#1: Function description}

The case study analyzes the emergency generated by the loss of propane gas during the transfer from tanker to tank. The objective of the model is to evaluate the variability of performance between upstream activities and downstream activities. Table 15 describes the activities carried out during the emergency and the responsibilities. The data are acquired from the internal company emergency plan.

Table 15: Functions of the system.

\begin{tabular}{|c|c|}
\hline Operation & Responsible \\
\hline $\begin{array}{c}1 \text { Activate "ESD" control from the control room to stop the transfer and close the } \\
\text { cut-off valves }\end{array}$ & Desk operator \\
\hline 2 Activate the shower cooling system on the truck through the $10 \mathrm{HC} 1 \mathrm{~V}$ system & Desk operator \\
\hline 3 If possible, intercept the ATB side valve & Driver \\
\hline 4 Disconnect the unloading arms & Internal operator \\
\hline 5 Turn away truck & Internal operator \\
\hline $\begin{array}{l}6 \text { Alternatively, continue the unloading operations until the tanker is emptied and } \\
\text { the gas is depressurized }\end{array}$ & Internal operator \\
\hline 7 Activate hydro-foam cannon & Internal operator \\
\hline 8 Turn off furnace and cool surrounding equipment & Internal operator \\
\hline
\end{tabular}

Figure 6 shows the FRAM of the emergency management activity. 


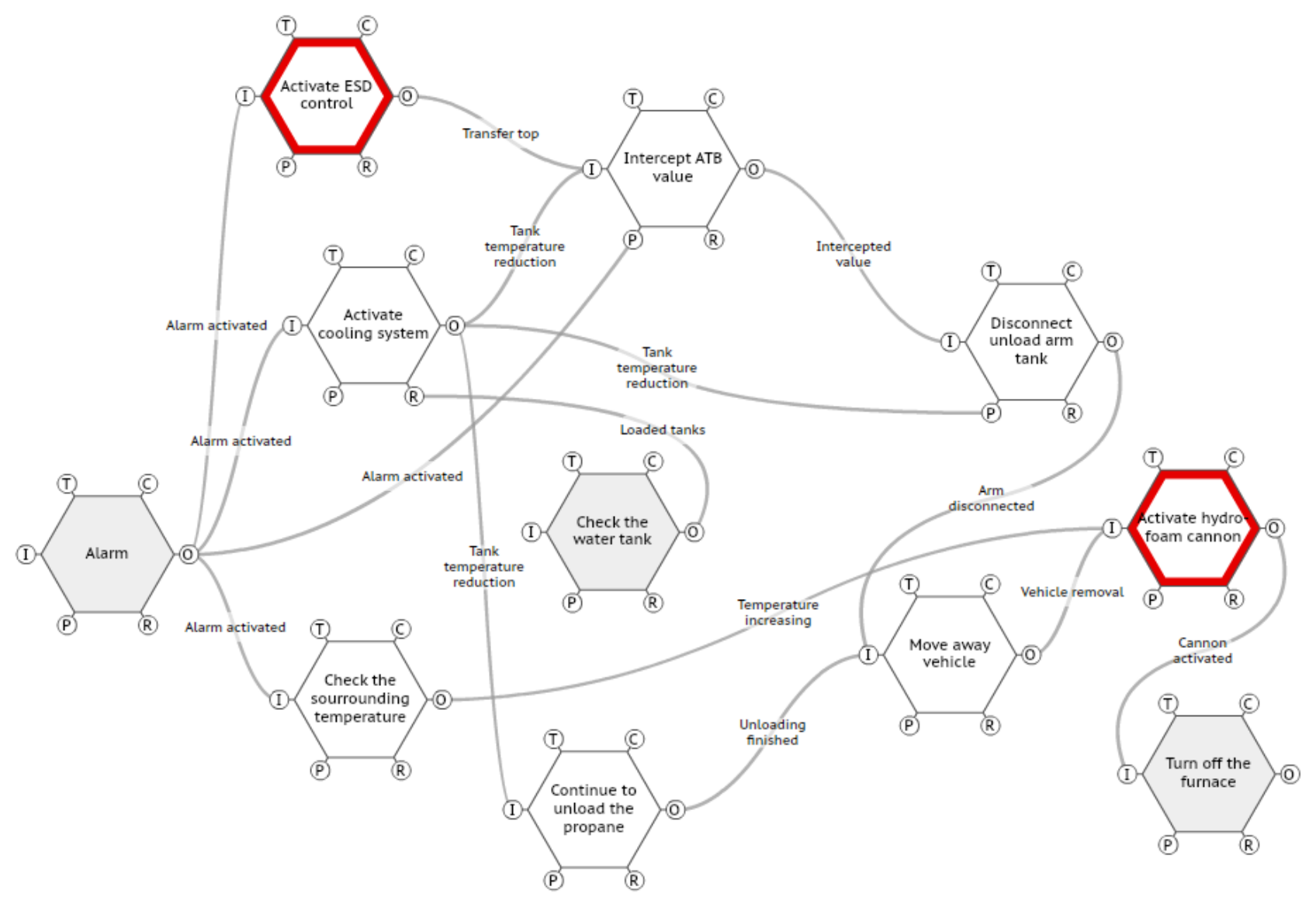

Figure 6: FRAM representation of the system.

\subsection{Step \#2: Function variability}

In the second step it is necessary to characterize the variability of the functions (in red in figure 6). The functions analyzed in the case study are human, which may present different variability. The analysis focuses on two main activities: "activate ESD control" and "activate hydro-foam cannon".

The analysis describes human functions, so it is essential to define the environmental conditions in which the operator is working. Tables 16 and 17 describe the environmental conditions in which the desk operator and the internal operator involved in the analyzed activities operate. The weights of the PSFs in relation to the functions are defined through interviews with the operators. The analysis describes the incident that occurred during the third hour of work, $\tau=2$.

Table 16: PSFs for "Activate ESD control".

\begin{tabular}{cccc}
\hline Factors & Level & Multipliers $(\boldsymbol{\alpha})$ & Weights $(\omega)$ \\
\hline Time & Nominal & 1 & 0.2 \\
Stress & High & 2 & 0.096 \\
Complexity & Medium High & 2 & 0.046 \\
Experience & High & 0.5 & 0.2 \\
Procedures & Poor & 5 & 0.34 \\
Ergonomics & Nominal & 1 & 0.075 \\
Process Work & Nominal & 1 & 0.043 \\
\hline
\end{tabular}


Table 17: PSFs for "Activate Hydro-foam cannon".

\begin{tabular}{cccc}
\hline Factors & Level & Multipliers $(\boldsymbol{\alpha})$ & Weights $(\boldsymbol{\omega})$ \\
\hline Time & Nominal & 1 & 0.075 \\
Stress & Extremely & 5 & 0.2 \\
Complexity & Nominal & 1 & 0.043 \\
Experience & Low & 3 & 0.2 \\
Procedures & Missing & 20 & 0.205 \\
Ergonomics & Poor & 10 & 0.132 \\
Process Work & Nominal & 1 & 0.145 \\
\hline
\end{tabular}

After defining the values of environmental factors, the relative weights and the factor $(\tau)$ which indicates the working time, it is possible to calculate the overall environmental index (OEI) associated to the two functions by applying equation 1.

- $\quad$ OEI (Activate ESD control) $=4.084$ (Scenario A);

- $\quad$ OEI (Activate Hydro-foam cannon) = 14.57 (Scenario B).

The results obtained show that two different scenarios are assumed for the two functions. For the activation of the ESD control scenario A is assumed, while for the activation of the hydro-foam gun, scenario B provides a greater variability of the function. For each scenario, it is necessary to identify a value of time variability and accuracy related to a specific function, each with its own score.

Considering the OEI values obtained, it is necessary to define the instance that is to be analyzed, identifying the output variability upstream for each function. In the case of the "Activate ESD control" function, an activity performed too early and a good accuracy of the activity is assumed (Table 18).

Table 18: Time and accuracy variability for "Activate ESD control".

\begin{tabular}{ccc}
\hline Output variability upstream function & Scores (a) \\
\hline Time & Too early & 2 \\
Accuracy & Accurate & 1 \\
\hline
\end{tabular}

In the case of the "Activate hydro-foam gun" function, it is assumed that the activity is too late and the accuracy of the activity acceptable (Table 19).

Table 19: For "Activate Hydro-foam cannon".

\begin{tabular}{ccc}
\hline \multicolumn{2}{c}{ Output variability upstream function } & Scores (a) \\
\hline Time & Too late & 3 \\
Accuracy & Acceptable & 2 \\
\hline
\end{tabular}

For each instance, it is necessary to identify the probability of occurrence. In the case study the time - precision dependence is very high $\mathrm{Q}=50 \%$. 
Considering time variability probability (shown in figures 4 and 5) it is possible to calculate the conditional probability precision - time (equation 2) for each function (Table 20).

Table 20: Probability of performance variability.

\begin{tabular}{ccc}
\hline & Prob (T) & Prob (A/T) \\
\hline Activate ESD Control & $15 \%$ & $16.70 \%$ \\
Activate Hydro-foam cannon & $25 \%$ & $41.70 \%$ \\
\hline
\end{tabular}

Finally, it is possible to calculate the total variability of the function (TV) for a given scenario (equation 4):

- $\quad$ TV (Activate ESD control) $=0.467$

- $\quad$ TV (Activate Hydro-foam cannon) $=1.434$

\subsection{Step \#3: Aggregation of variables}

For each function it is necessary to define the type of coupling upstream-downstream. The "Activate ESD control" function is connected to the "Intercept valve on the ATB side" function with an Output - Input connection. The "Activate hydro-foam cannon" function is also connected to the "turn off the furnace" function with an Output - Input link. Table 21 shows quantitative possible effects on downstream function.

Table 21: Function effects.

\begin{tabular}{|c|c|c|}
\hline $\begin{array}{l}\text { Output Variability of upstream } \\
\text { function }\end{array}$ & $\begin{array}{l}\text { Possible effects on downstream } \\
\text { function }\end{array}$ & $\operatorname{EF}(\mathbf{i}, \mathbf{j})$ \\
\hline Too early & Premature start $(\mathrm{V}+)$ & 2 \\
\hline Too late & Delayed activities $(\mathrm{V}+)$ & 2 \\
\hline Accurate & Possible damping (V-) & 0.5 \\
\hline Acceptable & No change $(\mathrm{V}=)$ & 1 \\
\hline
\end{tabular}

At this point it is possible to identify the variability of the performance (VAR) between the upstream function and the downstream function (equation 6):

- $\operatorname{VAR}($ Activate ESD control) $=0.467$

- $\operatorname{VAR}($ Activate Hydro-foam cannon) $=2.868$

\subsection{Step \#4: Monitoring and Management}

The FRAM analysis identified two main functions, realized during the management of an emergency in a production company. The model made it possible to calculate the performance variability generated on the upstream function that influences the downstream function. From the numerical analysis FRAM emerges a slightly critical value (considering the values shown in table $\mathrm{n}$ ) for the "activate hydro-foam cannon" function which must be analyzed to limit its variability, which affects the downstream variable. While the "Activate ESD control" function does not present a critical function variability for the downstream function.

In this study the focus is on the variability generated by external factors, therefore it is necessary to improve working conditions to limit the influences of environmental factors generating scenarios 
that determine a lower performance variability generated by the upstream function and also transmitted on the downstream function. In this case, to limit the variability of the critical function, it will be necessary to improve the procedures and reduce the complexity of the work, perhaps by developing standard work for the operators, which will help them during routine operations and emergency operations.

\section{Conclusions}

The increasingly complex nature of industrial plants and the need to analyze safety systems lead researchers to develop new models of analysis. Resilience engineering is one of the innovative models that allows to evaluate the performance variability of complex socio-technical systems. Among the various models of resilience engineering, the analysis focused on the FRAM, which represents the functions of a system and assesses possible weaknesses during daily activities.

Furthermore the FRAM allows to identify the critical functions for which the performance variability is extremely high and this could generate problems on the general system. If these situations occur, it is necessary to apply systems to dampen the performance variability.

This work exceeds the main gap of the traditional FRAM system: "the qualitative approach".

The research proposes the integration of the traditional FRAM with quantitative indicators that allow to evaluate the environmental influence on the operator and implements a Bayesian approach to evaluate the dependence between time and precision of an activity. The model was applied in a case study to evaluate the performance of emergency operations in a petrochemical company. Considering the variability of each system function, the paper numerically shows the level of variability generated by an upstream function on a downstream function. The results obtained are aimed at identifying particular functioncouplings that could generate high variability.

Future development of research is the integration of internal factors that influence the operator to be integrated with environmental factors to evaluate the variability of performance, also integrating technological and organizational aspects, beyond human ones. Moreover, the model can be applied in different socio-technical systems where a high level of complexity requires the use of innovative tools. This section is not mandatory, but can be added to the manuscript if the discussion is unusually long or complex.

Author Contributions: All authors contributed equally to this work.

Conflicts of Interest: The authors declare no conflict of interest.

\section{References}

1. Petrillo, A., Falcone, D., De Felice, F., \& Zomparelli, F. (2017a). Development of a risk analysis model to evaluate human error in industrial plants and in critical infrastructures. International Journal of Disaster Risk Reduction, 23, 15-24.

2. Casamirra, M., Castiglia, F., Corchia, L., Giardina, M., Lombardo, C., \& Messina, G. (2007, September). Risk analysis of the storage unit in a hydrogen refuelling station. In International Conference on Hydrogen Safety, San Sebastian.

3. Mechhoud, E. A., Rouainia, M., \& Rodriguez, M. (2016). A New tool for risk analysis and assessment in petrochemical plants. Alexandria Engineering Journal, 55(3), 2919-2931

4. Giardina, M., \& Morale, M. (2015). Safety study of an LNG regasification plant using an FMECA and HAZOP integrated methodology. Journal of Loss Prevention in the Process Industries, 35, 35-45

5. Zhou, X., Shi, Y., Deng, X., \& Deng, Y. (2017). D-DEMATEL: a new method to identify critical success factors in emergency management. Safety science, 91, 93-104.

6. Ramzali, Nahid, Mohammad Reza Miri Lavasani, and Jamal Ghodousi. Safety barriers analysis of offshore drilling system by employing Fuzzy Event Tree Analysis. Safety science 78 (2015): 49-59.

7. Akgün, İ., Gümüşbuğa, F., \& Tansel, B. (2015). Risk based facility location by using fault tree analysis in disaster management. Omega, 52, 168-179.

8. Hollnagel, E., 2012. FRAM: The Functional Resonance Analysis Method: Modelling Complex Socio-Technical Systems. Ashgate. 
9. De Felice, F., Zomparelli, F., \& Petrillo, A. (2017). Functional Human Reliability Analysis: A Systems Engineering Perspective. CEUR Workshop Proceedings 2010, pp. $23-29$

10. Rankin, A., Lundberg, J., Woltjer, R., Rollenhagen, C., \& Hollnagel, E. (2014). Resilience in everyday operations: a framework for analyzing adaptations in high-risk work. Journal of Cognitive Engineering and Decision Making, 8(1), 78-97.

11. Righi, A. W., Saurin, T. A., \& Wachs, P. (2015). A systematic literature review of resilience engineering: Research areas and a research agenda proposal. Reliability Engineering \& System Safety, 141, 142-152.

12. Bergström, J., van Winsen, R., \& Henriqson, E. (2015). On the rationale of resilience in the domain of safety: A literature review. Reliability Engineering \& System Safety, 141, 131-141.

13. Sawaragi, T., Horiguchi, Y., \& Hina, A. (2006, October). Safety analysis of systemic accidents triggered by performance deviation. In SICE-ICASE, 2006. International Joint Conference (pp. 1778-1781). IEEE.

14. Hollnagel, E., Pruchnicki, S., Woltjer, R., \& Etcher, S. (2008, April). Analysis of Comair flight 5191 with the functional resonance accident model. In 8th International Symposium of the Australian Aviation Psychology Association (pp. 8-pages).

15. Herrera, I. A., Hollnagel, E., \& Håbrekke, S. (2010, June). Proposing safety performance indicators for helicopter offshore on the Norwegian Continental Shelf. In PSAM 10-Tenth Conference on Probabilistic Safety Assessment and Management (p. 10).

16. Patriarca, R., Di Gravio, G., \& Costantino, F. (2016). Resilience engineering for space missions' safety assessment. In IAASS Proc. 8th IAASS Conf.'Safety First, Safety for all', ed. IAASS (Melbourne, Florida, 2016) (pp. 228-238).

17. Lundblad, K., Speziali, J., Woltjer, R., \& Lundberg, J. (2008, September). FRAM as a risk assessment method for nuclear fuel transportation. In Proceedings of the 4th International Conference Working on Safety (Vol. 1, pp. 223-1).

18. Shirali, G. A., Ebrahipour, V., \& Mohammd Salahi, L. (2013). Proactive risk assessment to identify emergent risks using functional resonance analysis method (fram): a case study in an oil process unit. Iran Occupational Health, 10(6).

19. Sujan, M. A., \& Felici, M. (2012). Combining failure mode and functional resonance analyses in healthcare settings. Computer safety, reliability, and security, 364-375.

20. Pereira, A. G. A. A. (2013). Introduction to the Use of FRAM on the Effectiveness Assessment of a Radiopharmaceutical Dispatches Process. In International Nuclear Atlantic Conference. Brazil.

21. Steen, R., \& Aven, T. (2011). A risk perspective suitable for resilience engineering. Safety science, 49(2), 292-297.

22. Belmonte, F., Schön, W., Heurley, L., \& Capel, R. (2011). Interdisciplinary safety analysis of complex socio-technological systems based on the functional resonance accident model: An application to railway trafficsupervision. Reliability Engineering \& System Safety, 96(2), 237-249.

23. Tian, J., Wu, J., Yang, Q., \& Zhao, T. (2016). FRAMA: a safety assessment approach based on Functional Resonance Analysis Method. Safety science, 85, 41-52.

24. Bjerga, T., Aven, T., \& Zio, E. (2016). Uncertainty treatment in risk analysis of complex systems: The cases of STAMP and FRAM. Reliability Engineering \& System Safety, 156, 203-209

25. Rosa, L. V., Haddad, A. N., \& de Carvalho, P. V. R. (2015). Assessing risk in sustainable construction using the Functional Resonance Analysis Method (FRAM). Cognition, Technology \& Work, 17(4), 559-573.

26. De Carvalho, P. V. R. (2011). The use of Functional Resonance Analysis Method (FRAM) in a mid-air collision to understand some characteristics of the air traffic management system resilience. Reliability Engineering \& System Safety, 96(11), 1482-1498.

27. Zheng, Z., Tian, J., \& Zhao, T. (2016). Refining operation guidelines with model-checking-aided FRAM to improve manufacturing processes: a case study for aeroengine blade forging. Cognition, Technology \& Work, 18(4), 777-791.

28. Praetorius, G., Graziano, A., Schröder-Hinrichs, J. U., \& Baldauf, M. (2017). FRAM in FSA - Introducing a Function-Based Approach to the Formal Safety Assessment Framework. In Advances in Human Aspects of Transportation (pp. 399-411). Springer International Publishing.

29. Patriarca, R., Di Gravio, G., \& Costantino, F. (2017). A Monte Carlo evolution of the Functional Resonance Analysis Method (FRAM) to assess performance variability in complex systems. Safety science, 91, 49-60.

30. Patriarca, R., Di Gravio, G., Costantino, F., \& Tronci, M. (2017). The Functional Resonance Analysis Method for a systemic risk based environmental auditing in a sinter plant: A semi-quantitative approach. Environmental Impact Assessment Review, 63, 72-86. 
31. Gertman, D., Blackman, H., Marble, J., Byers, J., \& Smith, C. (2005). The SPAR-H human reliability analysis method. US Nuclear Regulatory Commission.

32. Petrillo, A., De Felice, F., Falcone, D., Silvestri, A., \& Zomparelli, F. (2017). A Hybrid Probabilistic Model for Evaluating and Simulating Human Error in Industrial Emergency Conditions (HEIE). Journal of Failure Analysis and Prevention, 17(3), 462-476. 